Endoscopic Ultrasound (EUS) with tissue sampling has an established role in the diagnosis of pancreaticobiliary (PB) disease. Malignancy occurring following colonoscopy or gastroscopy that does not diagnose cancer is a recognised phenomenon and has been extensively investigated. There is little data on pancreatic cancer (PC) occurring following an EUS without evidence of cancer. We have studied the frequency, time course and potential risk factors for post EUS PC.

Methods PB EUS in England between Jan 2007 - Dec 2016 were identified in Hospital Episodes Statistics. Subjects with a diagnosis of PC made between 6 and 18 months after index PB-EUS were cases of post EUS PC. A logistic regression model examined risk factors for post EUS PC.

Results 79,490 PB EUS procedures were performed in 69,120 subjects, with 8,859 diagnosed with PC within 6 months of index EUS. $563(0.9 \%)$ subjects had post EUS PC (71\% 6 to 12 months and 29\% 12 to 18 months post EUS): median age was 70 (IQR 61-71) years and 57\% were male. The following factors were associated with post EUS PC: chronic pancreatitis (odds ratio 3.11(95\%CI 2.24-4.31), p<0.001); Charlson comorbidity score $>5$ (1.44 (1.04-1.98), $\mathrm{p}=0.028)$; and increasing age (1.01(1.00-1.02), $\mathrm{p}=0.005)$ Ethnicity, gender and EUS volume were not associated with post EUS PC (table 1). Post EUS PC rates varied in individual providers from: $1.0 \%$ in providers undertaking 8 to $111 \mathrm{~PB}$ EUS over the study period to $1.1 \%$ in providers undertaking 112 to $782 \mathrm{~PB}$ EUS and $0.7 \%$ in providers undertaking $>782$ PB EUS.

Conclusions $0.9 \%$ of subjects undergoing a PB EUS that did not diagnose PC were diagnosed with PC between 6 and 18

Abstract PTU-113 Table 1 Multiple Logistic Regression of factors associated with post EUS PC

\begin{tabular}{|c|c|c|c|c|}
\hline & Odds Ratio & $P$ value & $\begin{array}{l}\text { Lower } \\
95 \% \mathrm{Cl}\end{array}$ & $\begin{array}{l}\text { Upper } \\
95 \% \mathrm{Cl}\end{array}$ \\
\hline \multicolumn{5}{|l|}{ Age } \\
\hline Increasing years & 1.01 & 0.005 & 1.00 & 1.02 \\
\hline Sex & Baseline male & & & \\
\hline Female & 0.84 & 0.063 & 0.70 & 1.01 \\
\hline Deprivation Quintile & Baseline 1 & & & \\
\hline 2 & 0.88 & 0.409 & 0.66 & 1.18 \\
\hline 3 & 0.74 & 0.047 & 0.55 & 1.00 \\
\hline 4 & 0.82 & 0.175 & 0.62 & 1.09 \\
\hline 5 & 0.79 & 0.112 & 0.59 & 1.06 \\
\hline Unknown & 1.00 & & & \\
\hline Ethnicity & Baseline White & & & \\
\hline Asian & 1.13 & 0.688 & 0.62 & 2.05 \\
\hline Black & 1.29 & 0.472 & 0.65 & 2.58 \\
\hline Mixed & 0.56 & 0.564 & 0.08 & 4.08 \\
\hline Other & 2.13 & 0.004 & 1.27 & 3.57 \\
\hline Unknown & 15.01 & 0.000 & 11.78 & 19.14 \\
\hline Charlson score & Baseline 0 & & & \\
\hline $1-5$ & 1.15 & 0.235 & 0.91 & 1.46 \\
\hline$>5$ & 1.44 & 0.028 & 1.04 & 1.98 \\
\hline $\begin{array}{l}\text { Chronic pancreatitis } \\
\text { at index EUS }\end{array}$ & Baseline No & & & \\
\hline Yes & 3.11 & 0.000 & 2.24 & 4.32 \\
\hline Centre volume tertile & \multicolumn{4}{|c|}{ Baseline > 782 (Highest volume) } \\
\hline$<8$ & 4.54 & 0.171 & 0.52 & 39.56 \\
\hline 8-111 & 3.15 & 0.268 & 0.41 & 24.02 \\
\hline $112-782$ & 2.70 & 0.337 & 0.36 & 20.42 \\
\hline
\end{tabular}

months later. Post EUS PC was associated with chronic pancreatitis, co-morbidity and increasing age.

\section{PTU-114 LEARNING FROM ADVERSE EVENTS: A STUDY OF JAG ENDOSCOPY UNITS}

${ }^{1,2}$ Srivathsan Ravindran*, ${ }^{2}$ Hutan Ashrafian, ${ }^{3}$ Raphael Broughton, ${ }^{3}$ Michael Dron, ${ }^{3}$ Tim Shaw, ${ }^{2}$ Ara Darzi, ${ }^{1,2,3}$ Siwan Thomas-Gibson. 'St Mark's Hospital, London; ${ }^{2}$ Department of Surgery and Cancer, Imperial College, London; ${ }^{3}$ The Joint Advisory Group on GI Endoscopy (JAG), London

\subsection{6/gutjnl-2019-BSGAbstracts.103}

Introduction To meet JAG accreditation standards, endoscopy services are expected to have processes to identify, respond to and learn from adverse events (AEs). Units provide evidence to demonstrate they have met the set standard through audits of mortality and readmission after endoscopy. We conducted the first analysis of UK-wide learning outcomes from AEs based on mortality and readmission data. The aims were to understand how evidence is collected, describe relevant learning outcomes and resultant actions and processes used to share learning.

Methods A retrospective analysis of JAG 30-day mortality and 8-day readmission evidence from 2013-2018 was undertaken, assessing methods of data collection and documentation of learning outcomes. Data from evidence files were extracted and thematically analysed to identify and categorise learning outcomes and action points. This study was approved by the JAG research committee.

Results Complete data was available for 59 units. 42 units (71\%) used the JAG audit proforma in providing evidence. Where no JAG proforma was utilised, data was sourced from other audit summaries (14\%), PowerPoint slides $(8 \%)$ or other tabulated data (7\%). 35 units (59\%) documented learning outcomes following readmission or mortality, with $85 \%$ stating that outcomes were discussed in a formalised meeting.

Learning outcomes and action points are summarised below:

\begin{tabular}{ll} 
Abstract PTU-114 Table 1 & \\
\hline Learning Outcomes & Action Points \\
\hline High-risk patients & Systems \\
1. Increasing numbers & - Updating referral pathways \\
- Case selection & - Updating vetting pathways \\
- Clear communication & - Review of bowel preparation \\
- Clear documentation & protocols \\
- Careful planning Pre-assessment & - Updating patient information \\
- Screening renal function & leaflets \\
- Screening patients with renal impairment & - Post-procedure safety-netting \\
- Clear instruction for bleeding risks Endoscopist & Training \\
factors & - Guidance updates \\
- Decision-making & - Upskilling \\
- Therapy & - Nurse education Improving AE \\
- Procedure cessation & Data \\
- Knowledge & - Real time data analysis \\
- Skills & - Increasing audit frequency \\
& Shared Learning \& Feedback \\
& - Face-to-face feedback \\
& - Email feedback \\
& - Promoting reflective practice \\
& Unit meetings \\
\hline & \\
&
\end{tabular}


Conclusions Learning outcomes centre around managing highrisk patients, pre-assessment and endoscopist factors. Developing systems and training are actions in direct response to learning outcomes. Refining data collection methods was identified as a way to improve learning from AEs. There were a variety of methods to disseminate learning and feedback to endoscopists but no discernible mechanisms to share learning between units were identified. There needs to be a more robust way of collecting and collating endoscopy AE data, with a focus on shared learning between services.

\section{PTU-115 TIMING OF ENDOSCOPY AND 30 DAY MORTALITY IN PATIENTS ADMITTED WITH VARICEAL HAEMORRHAGE}

Syazeddy Samani*, Devinder Kaur, Ka-kit Li. University Hospitals of Leicester NHS Trust, Leicester City, UK

\subsection{6/gutjnl-2019-BSGAbstracts. 104}

Introduction Variceal haemorrhage carries a high mortality and current UK guidelines recommend endoscopy within 24 hours of admission but this is based on low level of evidence. ${ }^{1,2,3}$ The aim of this evaluation is to assess the association between timing of endoscopy with 30 day mortality in patients admitted with upper gastrointestinal variceal haemorrhage (UGVH).

Methods A total of 77 cases were retrospectively identified with a diagnosis of UGVH from the 1st of January 2017 to the 31st of December 2017 across three hospital sites in one UK NHS trust. 53 cases were analysed. Patients who did not present with acute UGVH on admission were excluded. Timing of endoscopy was defined as acute (0-12 hours), early (12-24 hours), delayed (>24hours) and calculated from time of admission to time of completion of endoscopy. Outcome measured was 30 day mortality. Fisher's exact test was used for statistical analysis ( $p<0.05$ defined as statistically significant).

Results The mean age of this cohort was 60.5 years old with $62.3 \%(n=33)$ male and 37.7\% $(n=20)$ female. Mean and median shock index (SI) at time of presentation were 0.81 and 0.8 (IQR 0.6-0.9) respectively. 73.6\% $(\mathrm{n}=39)$ had procedures performed within 24 hours. 34\% $(n=18)$ had acute endoscopy, 39.6\% $(n=21)$ had early endoscopy and $26.4 \%$ $(n=14)$ had delayed endoscopy. The mean SI for those who had acute endoscopy was highest (0.89) compared to the early $(0.78)$ and delayed endoscopy (0.76) group with mean UKELD scores of 51.6, 52 and 52.5 respectively. Overall 30 day mortality was $15.1 \%(n=8)$. Mortality rates between each group were as follows: 1) Acute endoscopy- 5.6\% ( $n=1), 2)$ Early endoscopy- 19\% ( $n=4), 3)$ Delayed endoscopy- $21.4 \%$ $(n=3)$. Statistical analysis showed no association between the different groups and 30 day mortality (acute vs early $\mathrm{p}=0.3849$, acute vs delay $\mathrm{p}=0.3377$ ).

Conclusion Mortality rate was lowest in the acute endoscopy group although statistical analysis suggested no significant association between timing of endoscopy and 30 day mortality. One may argue potential benefits of acute endoscopy based on the higher mean SI indicating greater mortality risk but a bigger, prospective multi-centre study is required to show the optimal timing and impact that timing of endoscopy may have on mortality for this cohort.

\section{REFERENCE}

1. Chen PH, Chen WC, Hou MC, et al. Delayed endoscopy increases re-bleeding and mortality in patients with hematemesis and active esophageal variceal bleeding: a cohort study. J Hepatol 2012;57:1207-13.

2. Dhiraj Tripathi, Adrian J Staley, Peter C Hayes, et al. UK guidelines on the management of variceal haemorrhage in cirrhotic patients. Gut 2015;0:1-25.

3. NICE CG141 - Acute upper gastrointestinal bleeding in over 16s: management

\section{PTU-116 DELAYED POST SPHINCTEROTOMY BLEEDING AND MANAGEMENT - 4 YEAR SINGLE CENTRE EXPERIENCE}

Lavanya Shenbagaraj ${ }^{*}$, Jordan White, Marek Czajkowski, Miles Allison. Department of Gastroenterology, Royal Gwent Hospital, Aneurin Bevan University Health Board, Newport, UK

\subsection{6/gutjnl-2019-BSGAbstracts.105}

Introduction Bleeding from endoscopic sphincterotomy (ES) is an important complication of therapeutic ERCP. The frequency of post sphincterotomy bleeding is estimated at $0.3 \%$ to $2 \%$. Delayed bleeding can occur anytime from hours up to two weeks after ES. Although several studies have addressed the risk factors for bleeding after ES, there is less information specifically on delayed bleeding.

Aims This study examines factors that influence delayed post ES related bleeding, and reviews its management and outcomes.

Methods We reviewed the records of patients who underwent an OGD within 4 weeks of having an ERCP procedure performed by a gastroenterologist between 2015 to 2018 at the Royal Gwent and Nevill Hall hospitals.

Results Over a 4 year period, 39 patients had an OGD within 4 weeks after an ERCP procedure. Of these, 17 had experienced delayed post ES bleeding at a median of 6 days (range1-10). The frequency of delayed post ES bleeding in our centre was $1.8 \%$. Most were male $12 / 17(70 \%)$ and the mean age was 74 years (range 45-97). Patients presented with melaena (41\%), hematemesis (24\%), haematochezia(6\%) or melaena with hematemesis(30\%). Out of the 17 patients, three were on aspirin, two were on clopidogrel and three were on warfarin. One had thrombocytopenia and three had a prothrombin time more than 13 seconds. Two had chronic kidney disease and ischaemic heart disease of which one patient was on regular dialysis. Indications for ES were choledocholithiasis (76\%), cholangitis (12\%) and malignancy (12\%). Endotherapy was applied with the following modalities, singly or in combination: adrenaline injection(2 patients), adrenaline injection and heater probe(1 patient), adrenaline injection and hemospray(4 patients), endoscopic clips(1 patient), adrenaline injection and $\operatorname{clips}(2$ patients) and hemospray alone (1 patient). No endotherapy was offered in 6 patients and were managed conservatively. One re-bled in 24 hours and responded to repeat endotherapy with adrenaline injection and hemospray. Four failed endotherapy and needed angiographic embolization. There were no deaths.

Conclusion This study emphasizes that factors such as thrombocytopenia, antiplatelet drugs, anticoagulants and cholangitis confer an increased risk of delayed post sphincterotomy bleeding. Patients who undergo ERCP with sphincterotomy should be warned about the $1.8 \%$ risk of delayed bleeding. Current guidelines suggest that ES can be done safely in patients on 